\title{
Analysis on Missile-borne Bistatic Forward-looking SAR Imaging Perfomance
}

\author{
Qi Chen ${ }^{1,2, \text { a }}$, Xiangping $\mathrm{Li}^{1}$, Mingbo Zhu ${ }^{1}$ and Xiaohai Zou ${ }^{1,2}$ \\ ${ }^{1}$ Naval Aeronautical and Astronautical University, Department of Electronic and Information Engineering, 264001 \\ Yantai, China \\ ${ }^{2}$ CEMEE State Key Laboratory, 471003 Luoyang, China
}

\begin{abstract}
Synthetic aperture radar (SAR) has been gradually applied to missile guidance for its advantages of all-weather, all-time and long-distance capabilities. Nevertheless, missile-borne SAR can not image on the area in front of the missile track restricted by SAR working characteristics. Missile-borne forward-looking SAR will be realized through separated transmitter and receiver. Iso-range contours, iso-Doppler contours, ground resolution and azimuth resolution are used to analyze the performance of missile-borne bistatic forwardlooking SAR, as to provide the theoretical basis for its application.
\end{abstract}

\section{Introduction}

In modern war, missile-based precision guided weapons have been the key restrictor that determines the process and the result of the war, making lots of countries compete for the development on them ${ }^{[1]}$. Infrared guidance, television guidance and radar guidance are the traditional ways for missile guidance, among which radar guidance has a good application prospect for its all-weather and all-time capabilities ${ }^{[2-4]}$. Missile-borne SAR guidance can provide two-dimensional images with high resolution, which has a number of advantages over one-dimensional imaging guidance. However, SAR can not image on the area in front of the platform track limited by the inherent working characteristics. Thus missile-borne SAR can only be used in matching guidance through side-looking or squint-looking imaging rather than homing guidance, restricting its application scenarios. The emergence of bistatic SAR offers a new way to solve this problem ${ }^{[5-8]}$. Bistatic SAR realizes forwardlooking through separated transmitter and receiver, making up the inherent disadvantages of monostatic SAR. Iso-range contours, Iso-Doppler contours, ground resolution and azimuth resolution are used in this paper to research the feasibility of missile-borne bistatic forward-looking SAR.

\section{Geometric model}

The geometric model of missile-borne bistatic forward-looking SAR is shown in Figure 1.

\footnotetext{
${ }^{\mathrm{a}}$ Corresponding author : chenqiyt@163.com
} 


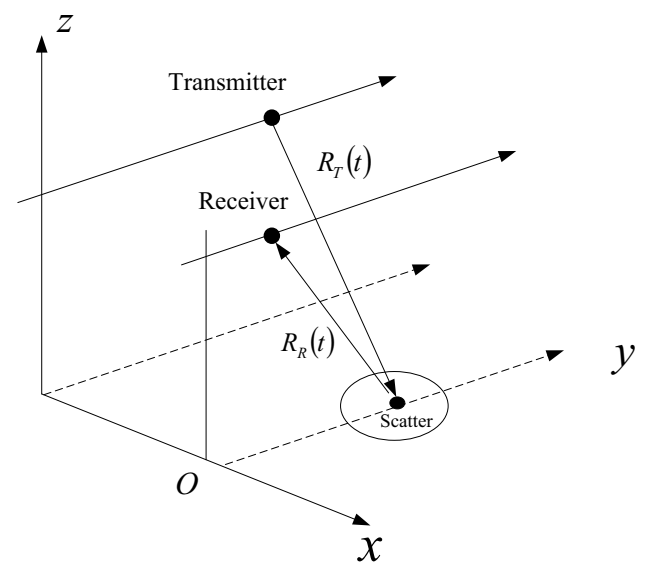

Figure 1. Geometric model of bistatic forward-looking SAR

The transmitter and receiver point together to the front area of receiver in the figure. The signals are transmitted to the target area in a certain PRT, then received and recorded by the receiver. $R_{T}(t)$ is the squint distance between the transmitter and the scatter, $R_{R}(t)$ is the squint distance between the receiver and the scatter.

\section{Iso-range contours and iso-Doppler contours}

Different scatters are distinguished through their distance to bistatic system in range direction. The range distance determines the time delay of the echo signal. The expression of range distance is

$$
R(t)=R_{T}(t)+R_{R}(t)
$$

Iso-range contours are composed of scatters with the same range distances, which are always in the form of ellipse.

The Doppler frequency of bistatic SAR is

$$
f=-\frac{1}{\lambda}\left(V_{T} I_{T}(t)+V_{R} I_{R}(t)\right)
$$

Where $V_{T}$ and $V_{R}$ are the velocities of the transmitter and receiver, respectively. $I_{T}(t)$ and $I_{R}(t)$ are the unit vector from the transmitter and receiver antenna phase centers to the scatter, respectively. IsoDoppler contours are composed of scatters with the same Doppler frequency.

SAR system can obtain two-dimensional image of the area where the iso-range contours and isoDoppler contours make rectangle or approximate rectangular partition.

When $V_{T}=V_{R}, R_{T}(t)=R_{R}(t)$, (3) and (4) refer to the calculation equation of monostatic SAR. The iso-range contours and iso-Doppler contours of an ordinary monostatic SAR system are shown in Figure 2. The arrow in the figure symbolizes the location and move direction of the SAR system. As is shown in the picture, the areas in side-looking and squint-looking direction have orthorhombic isorange contours and iso-Doppler contours, indicating the nice imaging performance in these areas. Meanwhile, the The approximatively parallel iso-range contours and iso-Doppler contours in forwardlooking direction account for the poor imaging performance in that areas. 


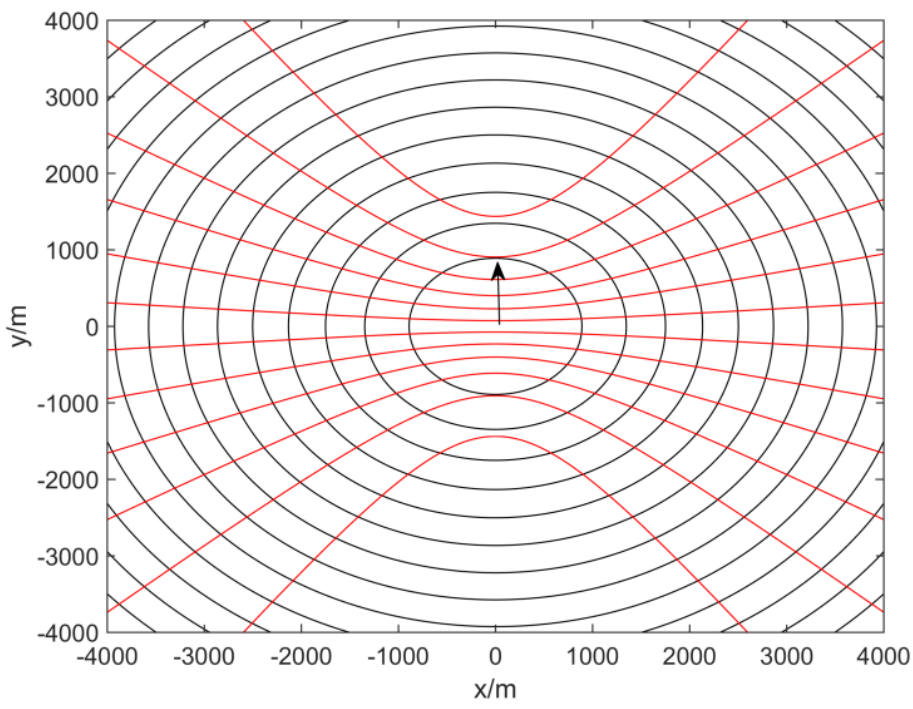

Figure 2. Monostatic SAR iso-range contours (circles) and iso-Doppler contours (lines)

\section{Ground resolution and azimuth resolution}

Derivated from the gradient method, the expression of bistatic SAR ground resolution is

$$
\rho_{r G}=\frac{c}{B \sqrt{2+2 \cos \beta-\left(\cos \phi_{T}+\cos \phi_{R}\right)^{2}}}
$$

Where $c$ is light velocity, $B$ is signal bandwidth, $\beta$ is bistatic angle, $\phi_{T}, \phi_{R}$ are the incidence angles of transmitter and receiver, respectively.

The expression of bistatic SAR azimuth resolution is

$$
\rho_{a}=\frac{D_{R}}{\sin ^{2} \theta_{R}+K_{V}^{2} / K_{R} \sin ^{2} \theta_{T}}
$$

Where $K_{V}$ is the transmitter to receiver velocity ratio, $K_{R}$ is the transmitter to receiver range distance ratio at center moment of synthetic aperture, $D_{R}$ is the azimuth size of receiver antenna, $\theta_{T}$ and $\theta_{R}$ are coangles of transmitter and receiver squint angle, respectively.

\section{Simulation and analysis}

Assume a bistatic SAR system in which the missile-borne transmitter is working on side-looking mode, the missile-borne receiver is working in forward-looking mode. Parameter Settings are shown in Table 1, iso-range contours and iso-Doppler contours are shown in Figure 3, distribution curves of ground resolution are shown in Figure 4 and distribution curves of azimuth resolution are shown in Figure 5. 
Table 1. Simulation parameters

\begin{tabular}{|c|c|}
\hline Parameters & Values \\
\hline Wavelength & $0.03 \mathrm{~m}$ \\
\hline Pulse bandwidth & $200 \mathrm{MHz}$ \\
\hline Pulse width & $2 \mu \mathrm{s}$ \\
\hline $\begin{array}{c}\text { Azimuth size of transmitter } \\
\text { and receiver antennas }\end{array}$ & $1 \mathrm{~m}$ \\
\hline PRF & $2500 \mathrm{~Hz}$ \\
\hline Transmitter velocity & $(0,500,0) \mathrm{m} / \mathrm{s}$ \\
\hline Receiver velocity & $(0,500,0) \mathrm{m} / \mathrm{s}$ \\
\hline Initial position of transmitter & $(-6000,0,1000) \mathrm{m}$ \\
\hline Initial position of receiver & $(0,-6000,1000) \mathrm{m}$ \\
\hline
\end{tabular}

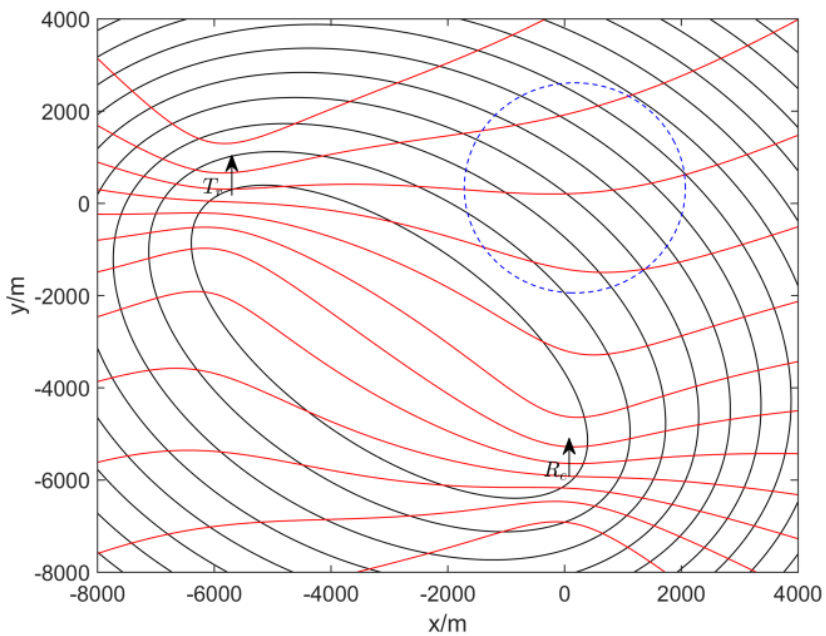

Figure 3. Bistatic SAR Iso-range contours (circles) and iso-Doppler contours (lines)

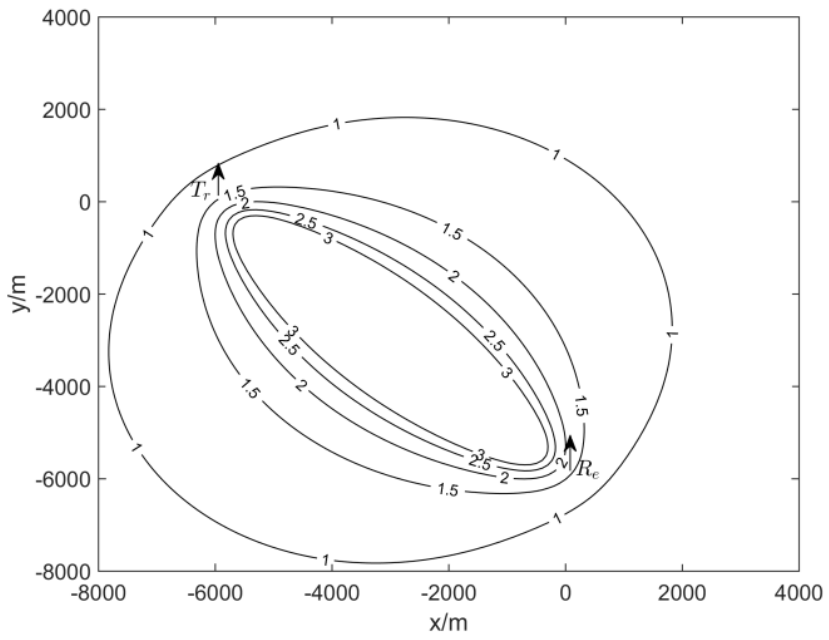

Figure 4. Ground resolution distribution curves 


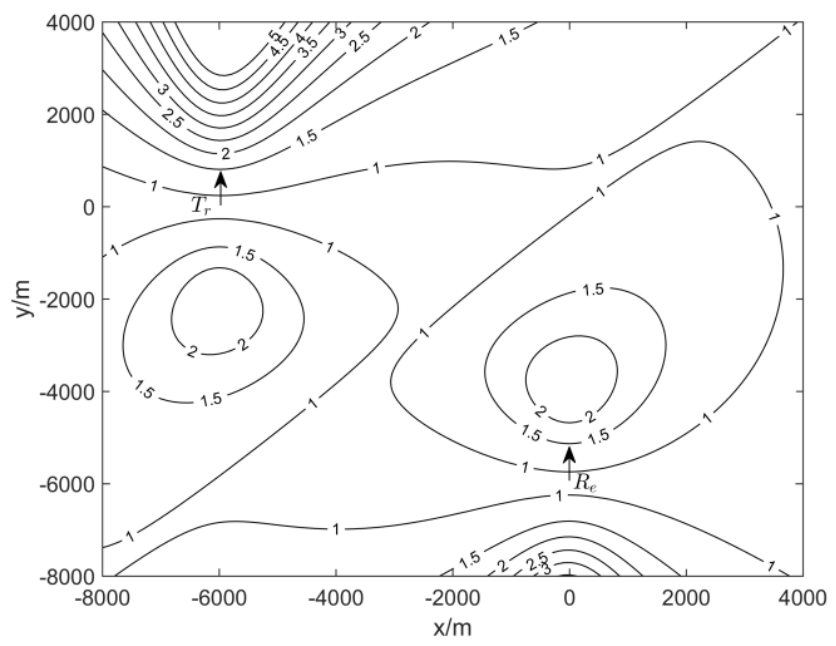

Figure 5. Azimuth resolution distribution curves

$T_{r}$ and $R_{e}$ are transmitter and receiver, respectively. The dashed part in Figure 3 is the imaging area. It is observed that the iso-range contours and iso-Doppler contours form rectangular segmentations in the imaging area, which indicates the forward-looking ability of the receiver. Therefore, the bistatic SAR system has high ground and azimuth solutions in the imaging area, as is shown in Figure 4 and Figure 5.

Echo simulation and imaging process are conducted for the point target at $(0,0,0)$. Point target simulation result is shown in Figure 6. The result tells the well focus in range and azimuth directions, which validates the correctness of the above conclusion.

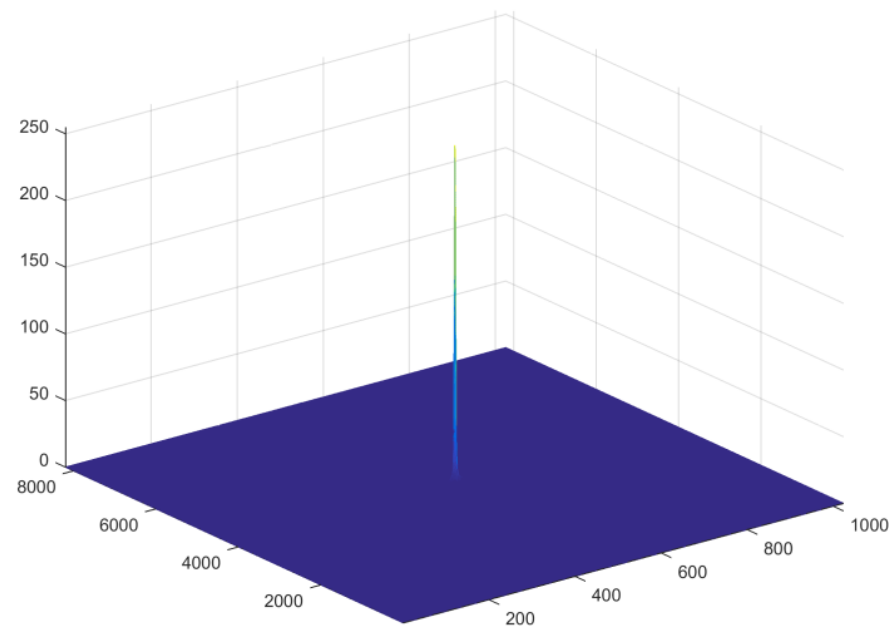

Figure 6. Simulation result of point target 


\section{Conclusions}

To verify the imaging performance of missile-borne bistatic forward-looking SAR, the iso-range contours and iso-Doppler contours of the system in which the transmitter is side-looking and the receiver is forward-looking are depicted. Then the ground and azimuth resolution calculation and point target simulation reveals the imaging ability of missile-borne bistatic forward-looking SAR. The results show that bistatic forward-looking SAR can be applied to missile platform. In practice, the transmitter can be placed on an UAV, a reconnaissance aircraft or a missile to illuminate along with the forward-looking missile-borne receiver to image the target area and realize forward-looking SAR guidance, which has a high application value.

\section{Acknowledgments}

This work is sponsored by open research foundation of State Key Laboratory (CEMEE2016K0201B).

\section{References}

1. H Wei, G Min. AMJ, 8, 56 (2016).

2. ZH Zhaorong, G He, ZH Xi, L Tao, K Yuhang. AMJ, 3, 23 (2016 ).

3. L Qi, Q Luyan. IT, 1, 146 ( 2016).

4. ZH Gang, ZH Mingbo, ZH Zhenbo, L Xiangping. AMJ, 9, 67 ( 2011).

5. Y Jianyu, H Yulin, Y Haiguang, W Junjie, L Wenchao, L Zhongyu, Y Xiaobo. IGARSS, 4202 (2013).

6. W Ingo, E Thomas, K Jens, R. B. Andreas, H. G. E. Joachim. IGARSS, 216 (2010).

7. E Thomas, W Ingo, K Jens, R. B. Andreas, H. G. E. Joachim. GRSL, 8, 765 (2011).

8. W Ingo, R. B. Andreas, K Jens. IGARSS, 327 (2012). 